\title{
Transparency in peer review
}

\author{
From December 2019, authors of research articles submitted to Nature Human Behaviour will have the option to \\ publish the full peer-review records of their manuscripts, including reviewer comments, editorial decision letters \\ and their own responses to reviewer and editorial comments.
}

$\mathrm{B}$ efore being published, our research manuscripts typically undergo two or three rounds of peer review. Peer reviewers invest a substantial amount of time and effort in reviewing the work of their peers, and they play a fundamental role both in evaluating the soundness of the work and in strengthening it for publication. The editors provide guidance on which reviewer comments are crucial to address, which are best left for future research or are unlikely to yield meaningful outcomes, and how the same standards of statistical analysis, reporting and interpretation can be upheld across all manuscripts. The authors provide detailed and thoughtful responses to reviewers and editors that accompany their revised manuscripts. The peer-review process is transformative for most of our manuscripts; however, this information has remained confidential to date.

This practice is now changing. Authors submitting their research manuscripts to Nature Human Behaviour from December 2019 onwards will have the opportunity to publish the full peer-review record associated with their manuscript, including editorial decision letters, peer-reviewer comments and author responses to reviewers and editors. Authors will be able to opt in to transparent peer review when an acceptance-in-principle decision is made on their manuscript. The peer-review record will not include confidential comments to the editors and will be edited to remove any confidential or third-party information. The remaining material will be published as is, without further editing.
Among the Nature Research journals, transparent peer review was first launched at Nature Communications in 2016; since then, more than $60 \%$ of authors have been opting in to transparent peer review at the journal. Nature Human Behaviour, along with six other Nature Research titles, is now joining Nature Communications in offering transparent peer review. Occasionally, our manuscripts are peer reviewed at a different Nature Research journal before being transferred to us. In those cases, unless the originating journal also offers transparent peer review, we will be unable to publish the peer-review record, and an editorial note will explain this in the published article.

If you receive an invitation to review one of our research manuscripts, the invitation will note clearly that, if the authors of the manuscript choose to opt in to transparent peer review, your comments will be published along with the manuscript. We hope that you will support this initiative by consenting to review and publicly share your comments. Although agreeing to review for us means agreeing that your comments will be published if the authors opt in to transparent peer review, your comments will still be anonymous by default. Your identity will remain confidential unless you choose to sign your review or let us know that you would like us to share your identity with the authors and our readers.

Nature Human Behaviour is a multidisciplinary journal, publishing research across the social, biomedical and physical sciences. We hope that transparent peer review will ultimately become the norm across the many disciplines we cover. However, we appreciate that peerreview conventions and expectations differ among disciplines. For instance, although single-blind peer review is the norm in the life sciences, double-blind peer review is the norm in some social sciences, such as political science. If, as an author, you choose not to publish the peer-review record associated with your manuscript, please do take a moment to let us know your reasons. If, as a reviewer, you choose to decline to review because you prefer that your comments not be made public, we would appreciate hearing your feedback. We hope that by actively demonstrating the value of transparent peer review, more and more scientists and communities will embrace it as a standard.

Opening up the peer-review process allows reviewers to be recognized and receive credit for their instrumental contributions to the publication process. Decision-making also becomes more transparent, and our editors can be held more accountable for our decisions. Authors have the opportunity to share more of the work behind their published article. In addition, transparent peer review benefits all readers, who have the opportunity to evaluate the quality of the peer-review process themselves. In adopting transparent peer review, we are taking a step towards supporting increased openness, accountability and trust in the publishing process.

Published online: 11 December 2019 https://doi.org/10.1038/s41562-019-0799-8 